\title{
Artroplastia Total da Anca: Estudo Retrospetivo e Comparativo entre a Abordagem Anterior Direta e a Abordagem Posterior
}

\section{Total Hip Arthroplasty: Retrospective and Comparative Study Between the Direct Anterior Approach and the Posterior Approach}

André Miguel Costaํ, José Pedro Fernandes², António Pedro Silva Eurico Bandeira Rodrigues ${ }^{1}$, Manuel Vieira da Silva ${ }^{1}$

Autor Correspondente: André Miguel Costa [andremdacosta@gmail.com] Avenida António Veiga n 163, 4740-405 Fão, Esposende, Portugal

\section{RESUMO}

INTRODUÇÃO: A procura constante pela melhoria levou ao aumento da popularidade das técnicas minimamente invasivas na artroplastia total da anca (PTA), como é o caso da abordagem anterior direta (AAD). Este estudo tem como objetivo a comparação, na PTA, dos resultados clínicos e funcionais entre a AAD e a abordagem posterior (AP), uma técnica muito otimizada.

MATERIAL E MÉTODOS: Foram incluídos 57 doentes submetidos a PTA pela mesma equipa cirúrgica. Avaliou-se a precisão da colocação do implante, o tempo de cirurgia, o tempo de internamento, as perdas sanguíneas, a dor durante o internamento, a recuperação funcional (WOMAC hip score) e a satisfação do doente.

RESULTADOS: As diferenças entre as abordagens não foram estatisticamente significativas na avaliação de parâmetros radiográficos de colocação dos implantes (offset global, ângulo acetabular e dismetria dos membros inferiores); apesar disso, a AAD demonstrou tendência para uma melhor precisão no ângulo acetabular. Também nesse grupo os doentes obtiveram um WOMAC hip score superior; um tempo de internamento, início do treino de marcha e tempo até abandonar as canadianas inferiores; no entanto, foram submetidos a tempos cirúrgicos superiores e sentiram maior dor durante o internamento. Não foram detetadas diferenças significativas na perda de sangue e satisfação do doente. DISCUSSÃO: Ambas as técnicas provaram ter um forte impacto na melhoria da qualidade de vida dos doentes. Os resultados vão de encontro aos de outros estudos, que referem uma maior precisão na colocação dos implantes, uma recuperação funcional mais rápida e sem complicações acrescidas para a AAD. $O$ adulto jovem é quem mais pode beneficiar desta abordagem.

PALAVRAS-CHAVE: Artroplastia Total da Anca; Osteoartrose da Anca; Qualidade de Vida

1. Serviço de Ortopedia e Traumatologia do Hospital de Braga, Braga, Portugal. 2. Serviço de Ortopedia e Traumatologia do Centro Hospitalar de São João, Porto, Portugal. Trabalho desenvolvido no Serviço de Ortopedia e Traumatologia do Hospital de Braga, Braga, Portugal.

Recebido: 03/01/2017 - Aceite: 07/03/2017 


\section{ABSTRACT}

INTRODUCTION: The constant search in total hip arthroplasty (THA) made popular minimally invasive technics like hip direct anterior approach (DAA). The aim of this study was to compare clinical and functional outcomes between DAA and posterior approach (PA) in THA.

MATERIAL AND METHODS: A total of 57 patients submitted to THA by the same surgical team were selected. Criteria like position of the implant, surgery duration, hospital stay, blood loss, post-operative pain, functional recovery (WOMAC hip score) and patient satisfaction were evaluated.

RESULTS: Regarding placement of the implant, the differences between both approaches were not statistically significant in all parameters (global offset, femoral-acetabular angle, leg length discrepancy). However, the DAA showed tendency for better acetabular angle results. This group had better WOMAC hip score; lower length of hospital stay, faster functional recovery and less amount of time needed to leave the crutches; nevertheless, more pain during post-operative period and increased surgery time were found. We found no significant differences in blood loss and patient satisfaction.

CONCLUSIONS: Both approaches improved patients' quality of life. These results confirm previous reports, with better implant placement, faster functional recovery and no further complications for DAA approach. A youngest patient may be the best candidate for this approach.

KEYWORDS: Arthroplasty, Replacement, Hip; Osteoarthritis, Hip; Quality of Life

\section{INTRODUÇÃO}

A artroplastia total da anca (PTA) é uma das técnicas cirúrgicas com maior sucesso na ortopedia. ${ }^{1,2}$ A maioria dos doentes submetidos a este procedimento testemunha, alívio da dor, aumento da mobilidade e da função e a retoma da qualidade de vida. Uma minoria de doentes continua a experienciar alguns sintomas, como a dor, que impede o regresso em pleno às atividades da vida diária (AVD). As possíveis causas apontadas são a infeção, a falência da fixação, a instabilidade articular e a lesão dos tecidos moles inerente ao procedimento cirúrgico. ${ }^{3}$ Como resposta a este problema, a última década ficou marcada pelo aumento da popularidade das técnicas minimamente invasivas na PTA. Algumas delas com taxas de complicações superiores às abordagens standard. ${ }^{4} \mathrm{~A}$ abordagem anterior direta (AAD) é uma modificação da abordagem anterior de Smith-Peterson, caracterizando-se pela progressão através de um plano intermuscular e internervoso, preservando os tecidos moles. ${ }^{5}$ São descritos benefícios na colocação dos componentes, ${ }^{6,7}$ nas perdas sanguíneas, ${ }^{6,8,9}$ na dor pós-operatória, ${ }^{1,6,7,10}$ na recuperação funcional ${ }^{2,11,12}$ e estadia hospitalar. ${ }^{1,6,10}$ Contudo, ainda não existe consenso final quanto aos reais benefícios desta abordagem - vários estudos revelam não existir qualquer vantagem ${ }^{13-15}$ e outros referem até piores resultados e mais complicações em relação à abordagem tradicional..$^{4,15-17}$ Apesar da controvérsia, a AAD é já uma técnica amplamente utilizada em todo o mundo, sendo importante esclarecer as suas vantagens. Neste estudo retrospetivo, comparamos a AAD com a abordagem posterior (AP), realizadas pela mesma equipa cirúrgica.
Os objetivos do estudo são avaliar os parâmetros radiológicos de colocação dos implantes, os parâmetros clínicos, os tempos cirúrgicos e de internamento, a recuperação funcional e o nível de satisfação do doente para comparação da AAD com a AP na artroplastia total da anca.

\section{MATERIAL E MÉTODOS}

Foram analisados os doentes submetidos a PTA pela mesma equipa cirúrgica; com classificação do estado físico ASA (American Society of Anesthesiologists) I ou II; e com diagnóstico inicial de osteoartrose primária da anca ou secundária a necrose avascular da cabeça femoral. Foi considerado o período compreendido entre janeiro de 2013 e dezembro de 2014. Estes foram os primeiros dois anos em que se realizou a AAD no nosso serviço.

A amostra foi dividida mediante a abordagem. Preferencialmente, doentes com idade $<60$ anos foram submetidos a $A A D$ e com idade $\geq 60$ anos foram submetidos a AP. Critérios como o sexo, idade, peso e marca de componentes foram avaliados, mas não selecionados como critérios de exclusão.

Todos os doentes realizaram o protocolo standard de pré e pós-operatório do nosso hospital. Os doentes submetidos a AP foram alvo de alguns ensinamentos como não cruzar as pernas, não fazer rotação interna do membro, não sentar em cadeiras baixas, usar elevador do tampo da sanita e dormir com uma almofada entre os membros inferiores. Aos doentes da AAD não foi dada qualquer restrição específica. 


\section{TÉCNICAS CIRÚRGICAS}

A AAD foi executada de acordo com o protocolo descrito noutros estudos..$^{18}$ Todos os doentes foram submetidos a anestesia geral e colocados em decúbito dorsal numa mesa cirúrgica, com os membros inferiores livres permitindo a sua mobilização, incluindo a hiperextensão da anca. A incisão inicia-se num ponto localizado $2-3 \mathrm{~cm}$ distalmente e 1-2 cm lateralmente à espinha ilíaca ântero-superior, estendendo-se distalmente na direção da cabeça do perónio, num total de 6-9 cm. Desenvolve-se um plano entre o músculo sartório e o músculo tensor da fáscia lata, procedendo-se depois à abertura da cápsula articular na sua região anterior. A osteotomia do colo do fémur permite a remoção da cabeça femoral e a preparação acetabular. A hiperextensão, adução e rotação externa do membro, permitem a exposição do fémur pela sua anteriorização. A colocação dos componentes é avaliada intraoperatoriamente com recurso ao fluoroscópio.

A abordagem posterior foi realizada de acordo com o já descrito. 19,20 Todos receberam indução anestésica epidural, sendo o doente posicionado em decúbito lateral. A incisão de 10-15 cm é realizada no aspeto posterior do grande trocânter com orientação na direção da cabeça do perónio. A fáscia lata é dissecada e as fibras do músculo glúteo máximo divididas proximalmente. A rotação interna da anca permite a exposição dos músculos rotadores externos que são sacrificados. Após a capsulotomia, a anca é luxada em flexão, adução e rotação interna. A osteotomia do colo do fémur permite preparar o acetábulo e o fémur de seguida.

\section{PARÂMETROS RADIOGRÁFICOS}

A avaliação radiográfica foi feita com base na radiografia ântero-posterior da bacia. O membro operado foi com-

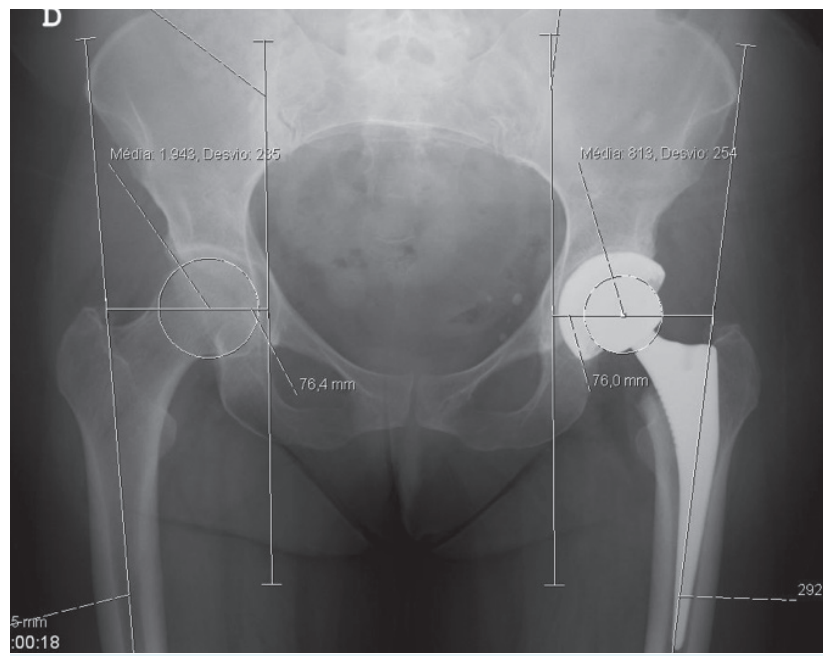

FIGURA 1. Medição do offset global. parado com o contralateral, exceto em situações de artroplastia bilateral, em que a comparação foi feita com a última radiografia prévia ao primeiro implante. Todas as medidas foram executadas por um investigador experiente. Foram avaliados os seguintes parâmetros: offset global - distância, em milímetros, medida desde o eixo longitudinal do fémur até à linha vertical tangente à vertente medial da lágrima da pélvis, atravessando o centro da cabeça femoral ${ }^{21}$ (Fig. 1); ângulo acetabular - medido entre a linha tangente aos bordos acetabulares e a linha transisquial22 (Fig. 2); e dismetria dos membros inferiores - distância, em milímetros, medida desde a vertente distal da lágrima até à linha que une os pontos mais largos dos pequenos trocânteres ${ }^{23}$ (Fig. 3).

\section{PARÂMETROS PERI-OPERATÓRIOS}

Foram consultados todos os registos efetuados pela equipa anestésica para cada ato cirúrgico. Avaliado o tempo cirúrgico e anestésico.

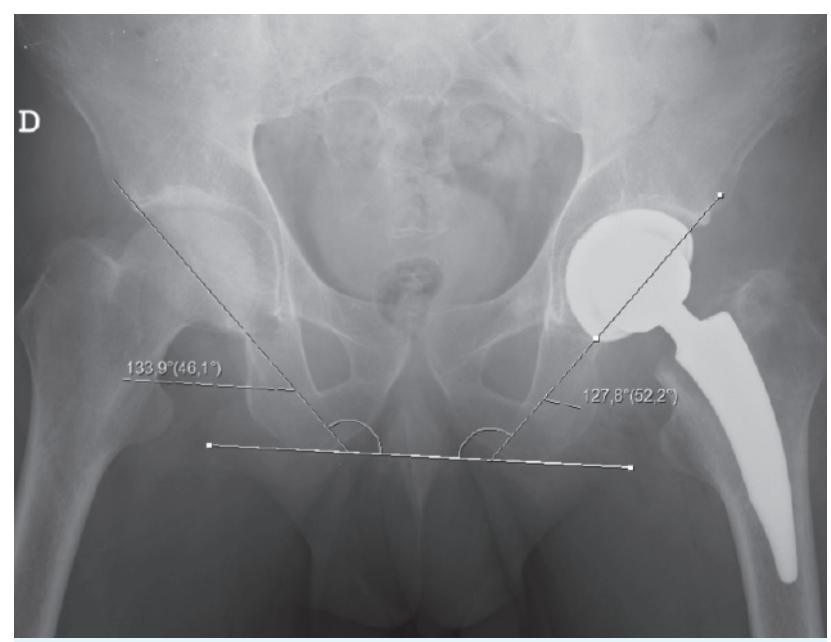

FIGURA 2. Medição do ângulo acetabular.

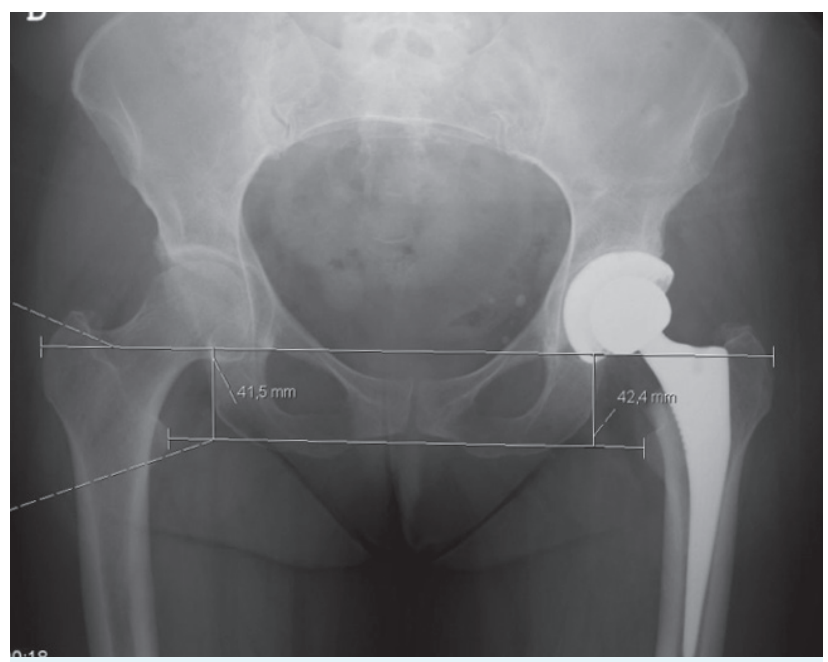

FIGURA 3. Medição da dismetria. 


\section{PARÂMETROS PÓS-OPERATÓRIOS}

Os dados relativos ao internamento foram retirados do diário clínico, tendo os registos sido efetuados pela equipa de ortopedia e de enfermagem responsáveis. Recolheram-se os seguintes dados: dor média no internamento - registo diário do nível de dor avaliada pela Escala Visual Analógica, com cálculo do valor médio para cada doente; perdas sanguíneas - diferença entre o valor de hemoglobina pré-operatória e o valor de hemoglobina no pós-operatório imediato, com exclusão dos doentes submetidos a transfusão nesse período; dia de início do treino de marcha, retirado do diário clínico, com exclusão dos doentes sem esta informação; tempo de internamento - nos doentes a aguardar disponibilidade de cuidadores, foi considerada a data em que o doente tinha condições clínicas para ter alta hospitalar; e complicações - luxações, fraturas, infeções, deiscência de ferida, dismetria dos membros inferiores e lesões neurovasculares.

\section{PARÂMETROS FUNCIONAIS}

Os dados relativos à recuperação funcional do doente foram recolhidos por contacto telefónico. Os números de telefone dos doentes foram aleatorizados e o contacto feito pelo investigador principal de forma cega. Foram excluídos os doentes em que não foi possível realizar a chamada telefónica. Os dados avaliados foram os seguintes: Western Ontario and McMaster Universities Arthritis Index (WOMAC hip score); tempo de uso de ortótese para auxílio da marcha - período, em semanas, que o doente necessitou de ortótese; satisfação com o resultado final da artroplastia - resposta afirmativa ou negativa; e satisfação com a cicatriz - resposta afirmativa ou negativa.

\section{ANÁLISE ESTATÍSTICA}

Toda a análise estatística foi feita no software IBM Statistical Package for the Social Sciences ${ }^{\circledR}$ versão 22.0 (SPSS). Para a análise das diferenças entre grupos, quando respeitados os princípios da normalidade (teste Kolomogorov-Smirnov), foram utilizados os testes paramétricos (Independent $t$ test e ANOVA). Recorreu-se, ainda, ao teste $d$ de Cohen para avaliação de impacto e aos Post-HOCs Tukey HSD para especificação das diferenças entre grupos. Nos casos em que a normalidade não se verificou, foram utilizados os seus equivalentes não paramétricos (Mann Whitney e Kruskal Wallis). A avaliação da associação entre variáveis categóricas foi feita através do teste Chi-square e Fisher exact test. Em todos os testes, um valor de $p$ inferior a 0,05 foi considerado estatisticamente significativo.

\section{ÉTICA}

As regras de conduta ética e de boas práticas foram acauteladas e o protocolo de estudo foi submetido e aprovado pela Subcomissão de Ética para as Ciências da Vida e da Saúde da Universidade do Minho (SECVS), pela Comissão de Ética para a Saúde do Hospital de Braga (CESHB) e autorizado pela Direção Clínica do Hospital de Braga.

\section{RESULTADOS}

Foram incluídos 57 doentes, 36 deles submetidos a artroplastia por AAD e 21 por AP. Nos resultados demográficos, o grupo AAD apresentou uma média de idade significativamente inferior à AP (48,9 vs 63,8 anos, p<0,001) (Tabela 1). Na AAD foram utilizados vários tipos de haste, ao contrário da AP, onde em apenas dois casos não foram utilizadas hastes standard. Para qualquer uma das abordagens, o diagnóstico mais prevalente foi a osteoartrose primária da anca (32 doentes na AAD e 20 na AP).

TABELA 1. Dados demográficos.

\begin{tabular}{|l|c|c|c|}
\hline Variável (média) & AAD & AP & $p$ \\
\hline Idade (anos) & 48,9 & 63,8 & $<0,001$ \\
\hline IMC $\left(\mathrm{kg} / \mathrm{m}^{2}\right)$ & 27,8 & 28,1 & 0,808 \\
\hline Masculino/Feminino & $24 / 9$ & $12 / 9$ & 0,236 \\
\hline
\end{tabular}

IMC - Índice de massa corporal; $p$ - valor de $p$.

Embora sem significado estatístico, a AAD demonstrou tendência para uma maior precisão ao nível do ângulo acetabular $\left(3,45^{\circ}\right.$ vs $\left.5,13^{\circ}, p=0,125\right)$ e da dismetria $(2,81 \mathrm{~mm}$ vs $3,51 \mathrm{~mm}, p=0,942$ ). No offset global, a AAD revelou-se ligeiramente menos precisa que a AP $(6,83 \mathrm{~mm}$ vs 6,29 $\mathrm{mm}, p=0,669)$, não sendo a diferença estatisticamente significativa (Tabela 2).

TABELA 2. Comparação das abordagens de acordo com os parâmetros radiográficos.

\begin{tabular}{|l|c|c|c|c|c|}
\hline & \multicolumn{4}{|c}{ AAD } & \multicolumn{3}{c|}{ AP } \\
\hline Variável & Média & DP & Média & DP & $p$ \\
\hline $\begin{array}{l}\text { Offset global } \\
(\mathrm{mm})\end{array}$ & 6,83 & 4,57 & 6,29 & 4,3 & 0,669 \\
\hline $\begin{array}{l}\text { Ângulo } \\
\text { acetabular }\left(^{\circ}\right)\end{array}$ & 3,45 & 2,5 & 5,13 & 4,44 & 0,125 \\
\hline $\begin{array}{l}\text { Dismetria } \\
(\mathrm{mm})\end{array}$ & 2,81 & 2,4 & 3,51 & 3,99 & 0,942 \\
\hline
\end{tabular}

DP - desvio padrão; $p$ - valor de $p$. 
TABELA 3. Comparação dos tipos de haste utilizados de acordo com os parâmetros radiográficos.

\begin{tabular}{|l|c|c|c|c|c|c|c|}
\hline & \multicolumn{2}{|c|}{ Sem preservação $(n=7)$} & Com preservação $(n=18)$ & \multicolumn{3}{c|}{ Standard $(n=32)$} \\
\hline Variável & Média & DP & Média & DP & Média & DP & $p$ \\
\hline Offset global $(\mathrm{mm})$ & 8,34 & 5,14 & 6,39 & 4,47 & 6,35 & 4,32 & 0,553 \\
\hline Dismetria $(\mathrm{mm})$ & 3,66 & 4,14 & 1,83 & 0,95 & 3,57 & 3,44 & 0,198 \\
\hline
\end{tabular}

DP - desvio padrão; $p$-valor de $p$.

Comparando os vários tipos de haste utilizados, não foram obtidos resultados com significado estatístico. $\mathrm{Na}$ dismetria, as hastes com preservação do colo femoral demonstraram tendência para valores mais próximos do fisiológico (1,83 mm, p=0,198). Ao nível do offset global, a haste com preservação e a standard apresentaram tendência para resultados melhores e muito aproximados (6,39 $\mathrm{mm}$ e 6,35 $\mathrm{mm}$ respetivamente, $p=0,553$ ) (Tabela 3).

Os doentes submetidos a AAD iniciaram o treino de marcha mais cedo ( 3,83 vs 5,29 dias, $p=0,001$ ), largaram as canadianas mais cedo $(6,33$ vs 9 semanas, $p=0,025)$ e tiveram um WOMAC score mais elevado (93,5 vs 82,9, $p=0,036)$. Por outro lado, os doentes da AP registaram níveis de dor inferiores durante o internamento (2,58 vs 2,1, $p=0,027)$. Embora sem significado estatístico, os doentes da AAD registaram uma tendência para menores perdas sanguíneas (2,87 vs 3,14 g/dL, p=0,349) e um mais rápido regresso às atividades de vida diárias (9,14 vs 11,6 semanas, $p=0,129$ ).

Os doentes submetidos a artroplastia por AAD necessitaram de internamentos mais curtos (6,27 vs 7,67 dias, $p=0,035)$. Contudo, o tempo cirúrgico na AP foi significativamente menor (68 vs $138 \mathrm{~min}, p<0,001$ ), assim como o tempo anestésico ( 88 vs 170 min, $p<0,001$ ). Foi possível constatar que com o aumento do número de artroplastias por AAD houve uma diminuição progressiva do tempo cirúrgico.

Até à data de realização do estudo não foi registada qualquer complicação nos doentes incluídos.

Qualquer que fosse a abordagem utilizada, mais de 90\% dos doentes afirmaram melhoria da qualidade de vida, satisfação com a cicatriz e satisfação com o resultado final, não se registando diferenças estatisticamente significativas entre as duas abordagens.

\section{DISCUSSÃO}

O número de PTA colocadas por AAD aumentou cerca de $20 \%$ nos últimos 10 anos. A base teórica para a sua utilização faz sentido para a maioria dos especialistas, sendo necessários, no entanto, estudos que determinem em quem deve ser aplicada e que comprovem completamente a sua superioridade. ${ }^{24}$
A melhoria sugerida pelos nossos resultados, sobretudo ao nível do ângulo acetabular na AAD, vai de encontro ao publicado anteriormente. ${ }^{6}$ A posição supina do doente durante a cirurgia possibilita a utilização facilitada do fluoroscópio, sendo este o principal fator descrito para esta diferença. ${ }^{25}$ Contudo, é necessária experiência na captação e interpretação das imagens durante a cirurgia, pois podem acontecer erros de avaliação radiográfica da cobertura acetabular. ${ }^{26}$

No que diz respeito à avaliação radiográfica da dismetria, optamos por um método de medição simples e com elevada fiabilidade. ${ }^{23}$

A rapidez da recuperação dos doentes do grupo AAD ficou bem patente nos nossos resultados. A melhoria funcional, o menor tempo de internamento e o regresso mais precoce às suas AVD, resultam numa diminuição dos custos hospitalares e aumento da produtividade. Outros estudos descreveram resultados semelhantes.1,2,6,10,11 Seria interessante perceber se estas vantagens se mantêm a longo prazo.

Contrariamente ao reportado noutros estudos, ${ }^{1,6,7,10} \mathrm{O}$ nosso grupo da AAD registou maior dor no pós-operatório em relação à AP, embora o valor médio seja relativamente baixo (2,58 em 10). A não execução de anestesia epidural no grupo da AAD será a principal justificação para esta diferença. A preferência pela anestesia geral neste grupo justifica-se com a necessidade de um maior grau de relaxamento muscular, pois nesta abordagem, ao contrário da AP, há uma total preservação muscular que dificulta a preparação óssea e a colocação dos implantes.

Resultados anteriores que testemunhavam uma diminuição da perda sanguínea com a $A A D^{6,8,9}$ foram encontrados na nossa amostra, mas sem relevo estatístico.

O maior número de complicações referido por alguns estudos ${ }^{4,16}$ também não foi detetado. Não foram registados, em nenhuma das abordagens, quaisquer casos de fratura, luxação, infeção, dismetria significativa, lesões neurovasculares ou outras.

Os tempos de anestesia e cirurgia foram substancialmente superiores na AAD, representando maior tempo para o doente sob o efeito da sedação e menor rentabilização do bloco. Alguns estudos referem a aproximação significativa dos tempos cirúrgicos entre abordagens, 
com o aumento da experiência e otimização da AAD.9,11 Apesar de não ter sido alvo de análise estatística, também foi possível perceber esta tendência no nosso caso.

Pela análise demográfica, foi clara a opção pela AAD em doentes mais jovens. Neste tipo de doente é preponderante preservar a anatomia e diminuir o tempo de inatividade, sendo vantagens das abordagens minimamente invasivas.1,3 Também nestes casos, a opção por hastes com preservação do colo femoral beneficiam o posicionamento do implante, podendo traduzir um melhor desempenho funcional a curto prazo. A qualidade do osso cortical do doente jovem permite, durante a artroplastia por AAD, a manipulação vigorosa do membro por parte do cirurgião, reduzindo a probabilidade de fraturas intraoperatórias. Atualmente, a cirurgia de revisão de uma PTA é realizada preferencialmente por AP e, desta forma, a AAD possibilita que a futura revisão ocorra numa área ainda não intervencionada.

Independentemente da abordagem, os doentes mostraram-se muito satisfeitos com o resultado final, ficando uma vez mais demonstrado o sucesso da PTA.

O estudo tem algumas limitações. $\bigcirc$ tempo de seguimento é curto e o tamanho da amostra limitado. A idade média dos dois grupos não é homogénea, podendo a idade menor dos doentes da AAD levar a uma recuperação funcional mais acelerada, a um menor tempo de internamento e a um regresso mais precoce às suas AVD. Os procedimentos anestésicos foram diferentes nos dois grupos, resultando em melhor controlo álgico nos doentes da AP através da anestesia epidural. Na AAD foram utilizados três tipos de haste femoral (haste curta sem preservação do colo, haste curta com preservação do colo e haste standard), enquanto na AP apenas em dois casos não foi utilizada a haste standard, pelo que este facto pode ter influência nos resultados.

Com esta investigação foi possível analisar a AAD desde o início da sua implementação no nosso hospital. Apesar de retrospetivo, o nosso estudo consegue analisar um grande número de variáveis, fornecendo informação importante para análise desta abordagem.

\section{CONCLUSÕES}

Ambas as técnicas provaram ter um forte impacto na melhoria da qualidade de vida do doente. A comparação da AAD com uma técnica já muito otimizada neste hospital pode diminuir as diferenças entre ambas. Contudo, o melhor resultado no WOMAC score e nos tempos de recuperação e a tendência para uma melhor precisão no ângulo acetabular representam vantagens significativas para esta técnica. A facilidade na utilização do fluoros- cópio também nos pareceu determinante, sendo uma mais-valia em relação à AP. É certo que as diferenças de idades dos nossos grupos podem estar na origem destas vantagens, mas, com isto, podemos concluir que a AAD representa uma alternativa sem complicações acrescidas para o adulto jovem, que fica menos tempo inativo. No idoso as vantagens não são tão óbvias, mantendo-se controversa a sua utilização.

CONFLITOS DE INTERESSE: Os autores declaram não ter qualquer conflito de interesse na realização do presente trabalho.

FONTES DE FINANCIAMENTO: Não houve qualquer fonte de financiamento na realização do presente trabalho.

CONFIDENCIALIDADE DOS DADOS: Os autores declaram ter seguido os protocolos da sua instituição acerca da publicação dos dados de doentes.

PROTEÇÃO DE PESSOAS E ANIMAIS: Os autores declaram que os procedimentos seguidos na elaboração do presente trabalho estão em conformidade com as normas das comissões de investigação clínica e de ética, bem como da declaração de Helsínquia e da AssociaçãoMédica Mundial.

\section{REFERÊNCIAS}

1. Berger RA, Jacobs JJ, Meneghini RM, Della Valle C, Paprosky W, Rosenberg AG. Rapid rehabilitation and recovery with minimally invasive total hip arthroplasty. Clin Orthop Relat Res. 2004;429:239-47.

2. Restrepo C, Parvizi J, Pour AE, Hozack WJ. Prospective randomized study of two surgical approaches for total hip arthroplasty. J Arthroplasty. 2010;25:671-9.

3. Bremer AK, Kalberer F, Pfirrmann CW, Dora C. Soft-tissue changes in hip abductor muscles and tendons after total hip replacement: comparison between the direct anterior and the transgluteal approaches. J Bone Joint Surg. 2011;93:886-9.

4. Spaans AJ, van den Hout JA, Bolder SB. High complication rate in the early experience of minimally invasive total hip arthroplasty by the direct anterior approach. Acta Orthop. 2012;83:342-6.

5. Unger AS, Stronach BM, Bergin PF, Nogler M. Direct anterior total hip arthroplasty. Instr Course Lect. 2014;63:227-38.

6. Schweppe ML, Seyler TM, Plate JF, Swenson RD, Lang JE. Does surgical approach in total hip arthroplasty affect rehabilitation, discharge disposition, and readmission rate? Surg Technol Int. 2013;23:219-27.

7. Barrett WP, Turner SE, Leopold JP. Prospective randomized study of direct anterior vs postero-lateral approach for total hip arthroplasty. J Arthroplasty. 2013;28:1634-8.

8. Parvizi J, Rasouli MR, Jaberi M, Chevrollier G, Vizzi S, Sharkey $\mathrm{PF}$, et al. Does the surgical approach in one stage bilateral total hip arthroplasty affect blood loss? Int Orthop. 2013;37:235762.

9. Chimento GF, Pavone V, Sharrock N, Kahn B, Cahill J, Sculco TP. Minimally invasive total hip arthroplasty: a prospective randomized study. J Arthroplasty. 2005;20:139-44. 
10. Alecci V, Valente M, Crucil M, Minerva M, Pellegrino CM, Sabbadini DD. Comparison of primary total hip replacements performed with a direct anterior approach versus the standard lateral approach: perioperative findings. J Orthop Traumatol. 2011;12:123-9.

11. Rodriguez JA, Deshmukh AJ, Rathod PA, Greiz ML, Deshmane PP, Hepinstall MS, et al. Does the direct anterior approach in THA offer faster rehabilitation and comparable safety to the posterior approach? Clin Orthop Relat Res. 2014;472:45563.

12. Nakata K, Nishikawa M, Yamamoto K, Hirota S, Yoshikawa H. A clinical comparative study of the direct anterior with mini-posterior approach: two consecutive series. J Arthroplasty. 2009;24:698-704.

13. Nam D, Sculco PK, Su EP, Alexiades MM, Figgie MP, Mayman DJ. Acetabular component positioning in primary THA via an anterior, posterolateral, or posterolateral-navigated surgical technique. Orthopedics. 2013;36:e1482-7.

14. Maffiuletti NA, Impellizzeri FM, Widler K, Bizzini M, Kain MS, Munzinger $U$, et al. Spatiotemporal parameters of gait after total hip replacement: anterior versus posterior approach. Orthop Clin North Am. 2009;40:407-15.

15. Ogonda L, Wilson R, Archbold P, Lawlor M, Humphreys P, O'Brien S, et al. A minimal-incision technique in total hip arthroplasty does not improve early postoperative outcomes. A prospective, randomized, controlled trial. J Bone Join Surg. 2005;87:701-10.

16. Bal BS, Haltom D, Aleto T, Barrett M. Early complications of primary total hip replacement performed with a two-incision minimally invasive technique. Surgical technique. J Bone Join Surg. 2006;88:221-33.
17. Woolson ST, Pouliot MA, Huddleston JI. Primary total hip arthroplasty using an anterior approach and a fracture table: short-term results from a community hospital. J Arthroplasty. 2009;24:999-1005.

18. Rachbauer F, Kain MS, Leunig M. The history of the anterior approach to the hip. Orthop Clin North Am. 2009;40:311-20.

19. Ranawat CS, Ranawat AS, Rasquinha VJ. Mastering the art of cemented femoral stem fixation. J Arthroplasty. 2004;19:8591.

20. Kelmanovich D, Parks ML, Sinha R, Macaulay W. Surgical approaches to total hip arthroplasty. J South Orthop Assoc. 2003;12: 90-4.

21. Lecerf G, Fessy MH, Philippot R, Massin P, Giraud F, Flecher $X$, et al. Femoral offset: anatomical concept, definition, assessment, implications for preoperative templating and hip arthroplasty. Orthop Traumatol Surg Res. 2009;95:210-9.

22. Woo RY, Morrey BF. Dislocations after total hip arthroplasty. J Bone J Surg. 1982;64:1295-306.

23. Meermans G, Malik A, Witt J, Haddad F. Preoperative radiographic assessment of limb-length discrepancy in total hip arthroplasty. Clin Orthop Relat Res. 2011;469:1677-82.

24. Chechik O, Khashan M, Lador R, Salai M, Amar E. Surgical approach and prosthesis fixation in hip arthroplasty world wide. Arch Orthop Trauma Surg. 2013;133:1595-600.

25. Masonis J, Thompson C, Odum S. Safe and accurate: learning the direct anterior total hip arthroplasty. Orthopedics. 2008;31(12 Suppl 2).

26. Alvarez AM, Suarez JC, Patel P, Benton EG. Fluoroscopic imaging of acetabular cup position during THA through a direct anterior approach. Orthopedics. 2013;36(10):776-7. 